\title{
Malignant transformation of the cultured human normal biliary tract epithelial cells induced by hepatitis $C$ virus core protein
}

\author{
RU-FU CHEN ${ }^{1}$, ZHI-HUA LI ${ }^{2}$, RAN-YI LIU ${ }^{4}$, XIAN-HE KONG ${ }^{3}$, QI-BIN TANG ${ }^{1}$ and JIE WANG ${ }^{1}$ \\ Departments of ${ }^{1}$ Hepatobiliary Surgery, ${ }^{2}$ Oncology and ${ }^{3}$ Digestion, The Affiliated Second Hospital, Sun Yat-sen University, \\ 107 Yan-Jiang Xi Road, Guang Zhou 510120; ${ }^{4}$ State Key Laboratory of Oncology in South China, Cancer Center, \\ Sun Yat-sen University, 51 Dongfeng Road East, Guang Zhou 510060, P.R. China
}

Received May 8, 2006; Accepted August 7, 2006

\begin{abstract}
High level expression of hepatitis $\mathrm{C}$ virus core protein (HCV-C) was detected in hilar cholangiocarcinoma tissues in our previous studies. This protein played an important role in the process of cancer cell inversion and proliferation, by some direct and indirect effects on certain genes. Based on this observation, we investigated the effect of HCV-C on human normal biliary epithelial (hBE) cell transformation and tumor development. Plasmid pHCV-C encoding the gene of $\mathrm{HCV}$ core protein was constructed and transfected into hBE cells. The expression and the bio-logical effect of HCV-C in HCV-C gene-modified hBE cells were determined in vitro and in vivo. The clone formation rates of hBE cells transfected with pHCV-C, pcDNA3.1 and mocktransfected cells were $36,2.5$ and $1.5 \%$, respectively. Tumor developed in 7 of 7 nude mice after incubated with pHCV-C transfected hBE cells, while no tumor appeared in mice injected with pcDNA3.1- and mock-transfected hBE cells. To investigate the possible mechanism of malignant transformation, we further studied the telomerase activity and human telomerase reverse transcriptase (hTERT) expression in pHCV-C transfected hBE cells. The elevated expression of hTERT was confirmed by RT-PCR, immunocytochemistry (ICC) and Western blot analysis, which in turn elevated the telomerase activity, confirmed by TRAP-ELISA. These results indicated that $\mathrm{HCV}-\mathrm{C}$ protein could participate in malignant
\end{abstract}

Correspondence to: Dr Qi-Bin Tang, Department of Hepatobiliary Surgery, The Affiliated Second Hospital, Sun Yat-sen University, 107 Yan-Jiang Xi Road, Guang Zhou 510120, P.R. China E-mail: tangqb@mail.sysu.edu.cn

Abbreviation: hBEC, human biliary epithelial cell; HCV, hepatitis $\mathrm{C}$ virus; $\mathrm{HCV}-\mathrm{C}$, hepatitis $\mathrm{C}$ virus core protein; H\&E staining, hematoxylin and eosin staining; hTERT, human telomerase reverse transcriptase; RT-PCR, reverse transcription-polymerase chain reaction; TRAP-ELISA, telomere repeat amplification protocol hybridization analysis

Key words: biliary, tumorigenesis, hepatitis C virus, telomerase transformation of human normal biliary epithelial cells and induce cholangiocarcinoma tumorigenesis, and the activation of telomerase was one of the possible mechanisms.

\section{Introduction}

$\mathrm{HCV}-\mathrm{C}$ gene encodes hepatitis $\mathrm{C}$ virus core protein, which possesses wide reverse activating effect (1). It has been validated that the expression of $\mathrm{HCV}-\mathrm{C}$ in transgenic mice induces hepatocellular fatty degeneration and development of hepatocellular carcinoma. Hence, HVC-C is considered to be one of the etiological factors of liver cancer (2).

Cholangiocarcinoma originated from the malignant transformation of biliary tract endothelial cells. High level expression of HCV-C in hilar cholangiocarcinoma tissues indicated that $\mathrm{HCV}-\mathrm{C}$ protein play an important role in the process of cancer cells inversion and proliferation, through some direct or indirect effects on certain oncogenes, tumor suppressor genes and cell apoptosis (3). However, in most of the pervious studies, cholangiocarcinoma cell lines, but not normal biliary tract endothelial cells, were used to investigate the mechanism of cholangiocarcinoma generation (4-6). In this study, a human normal biliary tract endothelial cell (hBEC) line was used to investigate the tumorigenesis ability of $\mathrm{HCV}-\mathrm{C}$ and its potential mechanism. Our data indicate that $\mathrm{HCV}-\mathrm{C}$ protein was able to induce malignant transformation of $\mathrm{hBE}$ cells, and the activation of telomerase was one of the possible mechanisms.

\section{Materials and methods}

Cell lines and animals. hBE cells is a human normal biliary tract endothelial cell line, kindly provided by Professor S.M. Cruickshank (7). QBC939 is a hilar cholangiocarcinoma cell line (8), kindly presented by Professor S.-G. Wang from the Third Military Medical University. Cells were cultured at $37^{\circ} \mathrm{C}$ in $5 \% \mathrm{CO}_{2}$ in DMEM/F12 medium supplemented with human recombinant epidermal growth factor $(10 \mu \mathrm{g} / 1$, Sigma), 3-iodina-L-thyronine (5 $\mu \mathrm{g} / \mathrm{l}$, Sigma), hydrocortisone (0.4 mg/l, Sigma), insulin (5 mg/l, Sigma), human recombinant hepatic cell growth factor $(5 \mu \mathrm{g} / \mathrm{l}$, Sigma) and $10 \%$ fetal bovine serum.

Nude Balb/C mice, male, aged 6-8 weeks and weighing 20-24 g, were used in this study. All animals were housed in an environmentally controlled facility with a 12 -h lighting. 
They were given free access to standard chow and water. All animals were handled in accordance with the Institutional Guidelines for the Care and Use of Laboratory Animals.

Plasmid construction. A cDNA clone (Blue4/C5p-1) of strain $\mathrm{HCV}-1$ was used as a template for amplification of the core region (amino acids 1-191) by PCR. Two synthetic oligonucleotide primers, (sense: 5'-CCGGATCCATGAGCACA AATCCT-3'; antisense: 5'-GGAAGCTTAGTGGAAA CTGGGACGG-3'), were used for PCR amplification by a procedure similar to Hiramatsu et al (9). The amplified DNA was inserted into the BamH I/Hind III site of the pcDNA3.1 mammalian expression vector (Invitrogen, San Diego, CA), constructing the HCV-C expressing plasmid pHCV-C. Subsequently, the DNA sequence of $\mathrm{HCV}$ core protein in pHCV-C was sequenced.

hBE cell transfection and screening. hBE cells were seeded in 6-well plates before transfection and incubated at $37^{\circ} \mathrm{C}$ in $5 \% \mathrm{CO}_{2}$. Five micrograms of plasmids pHCV-C was transfected into hBE cells with lipofectamine kit (Invitrogen), pcDNA3.1 as vector control. Stably expressing cells (hBEC$\mathrm{HCV}-\mathrm{C})$ were obtained after screening in culture medium with G418 $(800 \mu \mathrm{g} / \mathrm{ml})$ for three weeks. Growth morphology and characteristics were carefully observed.

RT-PCR analysis. RT-PCR was performed to examine the expression of HCV-C or hTERT. Total-RNA was isolated using TRIzol reagent (Invitrogen). RNase-free DNase (Promega) was used to eliminate the possibility of plasmid DNA contamination. Five micrograms of total-RNA was transcribed into cDNA by using AMV Reverse Transcriptase (Promega). Primers for HCV-C (sense: 5'-GCACAAATCCT AAACTC-3'; antisense: 5'-GGACGGTCAGACAGGACAG CA-3') and for hTERT (sense: 5'-CGGAAGAGTGTCTGGA GCAA-3'; antisense: 5'-GGATGAAGCGGAGTCTGGA-3') were used for PCR amplification. The amplification was initiated with $5 \mathrm{~min}$ of denaturation at $94^{\circ} \mathrm{C}$, followed by 30 cycles at $94^{\circ} \mathrm{C}$ for $1 \mathrm{~min}, 55^{\circ} \mathrm{C}$ for $1 \mathrm{~min}$ and $72^{\circ} \mathrm{C}$ for $90 \mathrm{sec}$. After the last cycle of amplification, the samples were incubated for $7 \mathrm{~min}$ at $72^{\circ} \mathrm{C}$. The PCR products were analyzed by ethidium bromide staining on $1.5 \%$ agarose gels.

Immunocytochemistry and immunohistochemistry. Immunocytochemistry (ICC) and immunohistochemistry (IHC) were performed to detect the expression of HCV-C using a Dako EnVision $^{\mathrm{TM}}$ System (Peroxidase). Mouse anti-HCV core antigen monoclonal antibody (Abcam plc.) was used as a primary antibody. Diaminobenzidine was used as the chromogen and cells were counterstained with hematoxylin.

Western blot analysis. Cell lysates were separated by SDSPAGE and transferred to a PVDF membrane. Blots were visualized with a Phototope-HRP Western blot detection system (New England BioLabs). Briefly, membranes were blocked with 5\% non-fat dry milk powder in TTBS $(20 \mathrm{mM}$ Tris- $\mathrm{HCl}$, $\mathrm{pH} 8.0,0.8 \% \mathrm{NaCl}, 0.1 \%$ Tween-20) overnight at $4^{\circ} \mathrm{C}$, followed by a 4-h incubation in the presence of corresponding antibodies for HCV-C or hTERT (1:1000 dilutions) at room temperature. Blots were rinsed with TTBS solution, and incubated with the secondary antibody, HRP-linked antimouse IgG (Santa Cruz, 1:1000) for $2 \mathrm{~h}$. After rinsing, blots were incubated with LumiGLO ${ }^{\text {TM }}$ (New England BioLabs) for $1 \mathrm{~min}$ and exposed to X-ray film.

Cell proliferation assay. hBEC-HCV-C cells, hBEC-pcDNA 3.1 cells and mock-transfected hBE cells were suspended and seeded in 6-well plate with a concentration of $2 \times 10^{5}$ per well. The cells were incubated at $37^{\circ} \mathrm{C}$ in $5 \% \mathrm{CO}_{2}$ in DMEM/ F12 medium for $24,36,48,60$ and $72 \mathrm{~h}$ separately, and then collected by trypsinization. Cells were counted under microscope by three researchers, respectively.

Soft agar assays. Soft agar dishes were prepared with an underlayer of $0.75 \%$ agarose (Sigma) in DMEM/F12 supplemented with $10 \%$ fetal calf serum. Cells were suspended and seeded in the dishes. The dishes were examined microscopically for colony formation after incubation for 14 days. The data were expressed as the percentage of colonies containing more than 50 cells 2 weeks after plating.

Tumorigenesis in vivo. Twenty-eight nude/BalbC mice were randomly divided into 4 groups. hBE cells, hBEC-pcDNA 3.1 cells, hBEC-HCV-C cells and QB939 cells $\left(5 \times 10^{6}\right)$ were subcutaneously injected to the flank of nude/BalbC mice, respectively. The body weight of mice and size of tumor were measured once a week. The mice were sacrificed 40 days later. The tumor tissues were removed, fixed with $4 \%$ formalin and paraffin embedded. H\&E staining and immunohistochemical staining were carried out.

TRAP-ELISA. The telomerase activity was examined by telomere repeat amplification protocol hybridization analysis. Briefly, the cells were collected by trypsinisation and washed twice with ice-cold PBS solution. Cells $\left(1 \times 10^{6}\right)$ were used for the following TRAP-ELISA assay, and the process was performed according to the introduction of TRAP-Hyb kit. Positive and negative control was set with those provided in the kit. The hybridization result was detected with ELX800, and the OD value was under $450 / 595 \mathrm{~nm}$. The OD value of negative control was calculated as 0.05 when its real level was $<0.05$. The positive result of telomerase activity was defined when the sample OD level was no less than 2.1 times that of negative control.

\section{Results}

Identification of $\mathrm{pHCV}-\mathrm{C}$. Plasmid $\mathrm{pHCV}-\mathrm{C}$ was digested with XhoI and HindIII. The size of the bands correlated to the expected size of the products, which were $580 \mathrm{bp}$ and $5.8 \mathrm{~kb}$, respectively (data not shown). Sequencing analysis showed that the sequence of cloned $\mathrm{HCV}-\mathrm{C}$ gene was the same as that published previous (GenBank Accession no. DQ071885).

$H C V-C$ expression in hBEC-HCV-C cells. HCV-C mRNAs were detected in $\mathrm{pHCV}-\mathrm{C}$ transfected hBE (hBEC-HCV-C) cells by RT-PCR analysis, while none were detected in pcDNA3.1-transfected hBE (hBEC-pcDNA3.1) cells or parent hBE cells (Fig. 1A). Western blot analysis showed the expression of HCV-C protein in hBEC-HCV-C cells, but not 
A

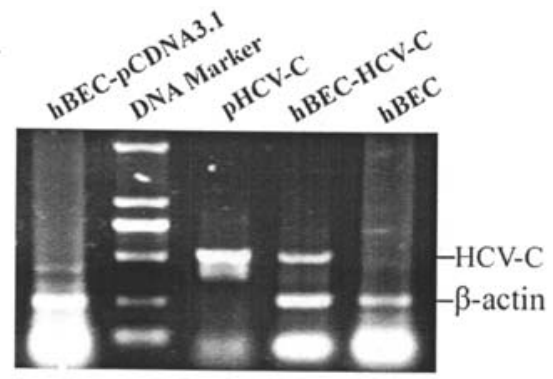

$\mathrm{B}$

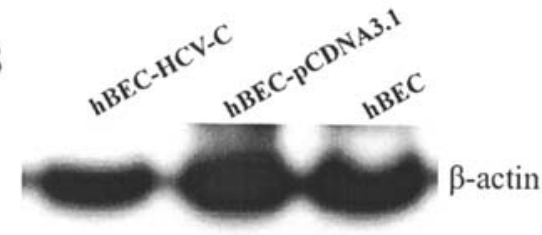

$\mathrm{HCV}-\mathrm{C}$

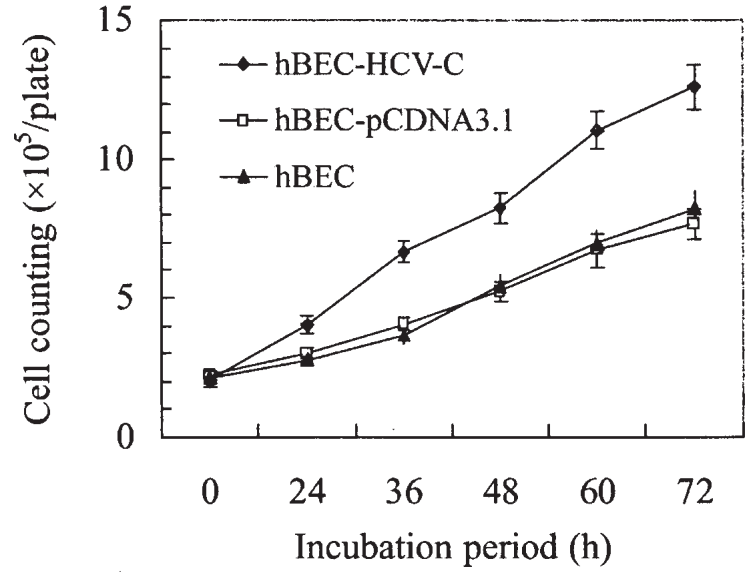

Figure 3. The effect of HCV-C gene transfection on hBE cells proliferation. hBEC-HCV-C cells, hBEC-pcDNA 3.1 cells and mock-transfected hBE cells were suspended and seeded in 6-well plate with a concentration of $2 \times 10^{5}$ per well. The cells were incubated at $37^{\circ} \mathrm{C}$ in $5 \% \mathrm{CO}_{2}$ in DMEM/F12 medium. Cells were counted 24, 36, 48, 60 and $72 \mathrm{~h}$ later.

Figure 1. HCV-C expression in pHCV-C transfected hBEC. (A), RT-PCR analysis of the expression of HCV-C antigen in transfected hBEC. Total cellular RNAs of pHCV-C transfected hBEC (hBEC-HCV-C) cells, pcDNA3.1-transfected hBEC (hBEC-pcDNA3.1) cells and mock-transfected hBEC were isolated, HCV-C mRNAs were detected by RT-PCR amplification, with $\beta$-actin used as the internal control (HCV-C $580 \mathrm{bp}$, ß-actin $417 \mathrm{bp}$ ). (B), Western blot analysis of the expression of HCV-C antigen in transfected hBEC.

in hBEC-pcDNA3.1 cells or hBE cells (Fig. 1B). Immunocytochemical analysis confirmed the expression of $\mathrm{HCV}-\mathrm{C}$ protein in hBEC-HCV-C cells, which located in the cellular plasma (Fig. 2). Morphological observation found that nucleus enlarged in $\mathrm{HCV}-\mathrm{C}$-positive hBE cells, indicating its malignant transformation.

Cell proliferation and clone formation. hBEC-HCV-C cells showed a higher proliferating ability than hBEC-pcDNA 3.1 cells and mock-transfected hBE cells. The doubling time of hBEC-HCV-C cells was $24.2 \mathrm{~h}$, while those of hBECpcDNA 3.1 cells and mock-transfected hBE cells were 39.6 and 39.9 h, respectively (Fig. 3). The results suggested the expression of $\mathrm{HCV}-\mathrm{C}$ could promote the proliferation of $\mathrm{hBE}$ cells and result in immortalization.
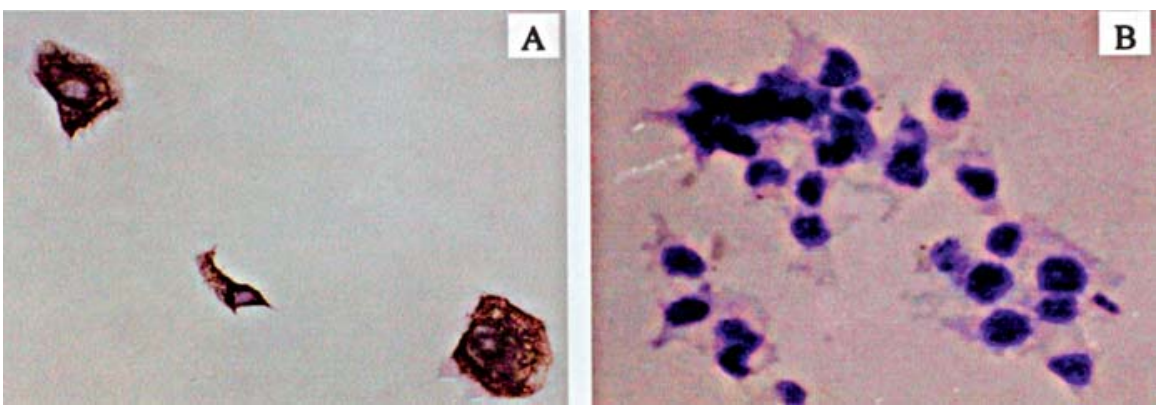

Figure 2. Immunocytochemical analysis of the expression of HCV-C antigen in transfected hBEC. hBE cells tranfected with pHCV-C (A) or pcDNA3.1 plasmid (B) were screened with G418 (800 $\mu \mathrm{g} / \mathrm{ml})$ for 3 weeks. The expression of HCV-C antigen was checked by immunocytochemistry. Mouse anti-HCV core antigen monoclonal antibody was used as a primary antibody. Diaminobenzidine was used as the chromogen and cells were counterstained with hematoxylin. Brown staining indicates the expression of HCV-C (ICC x400). 


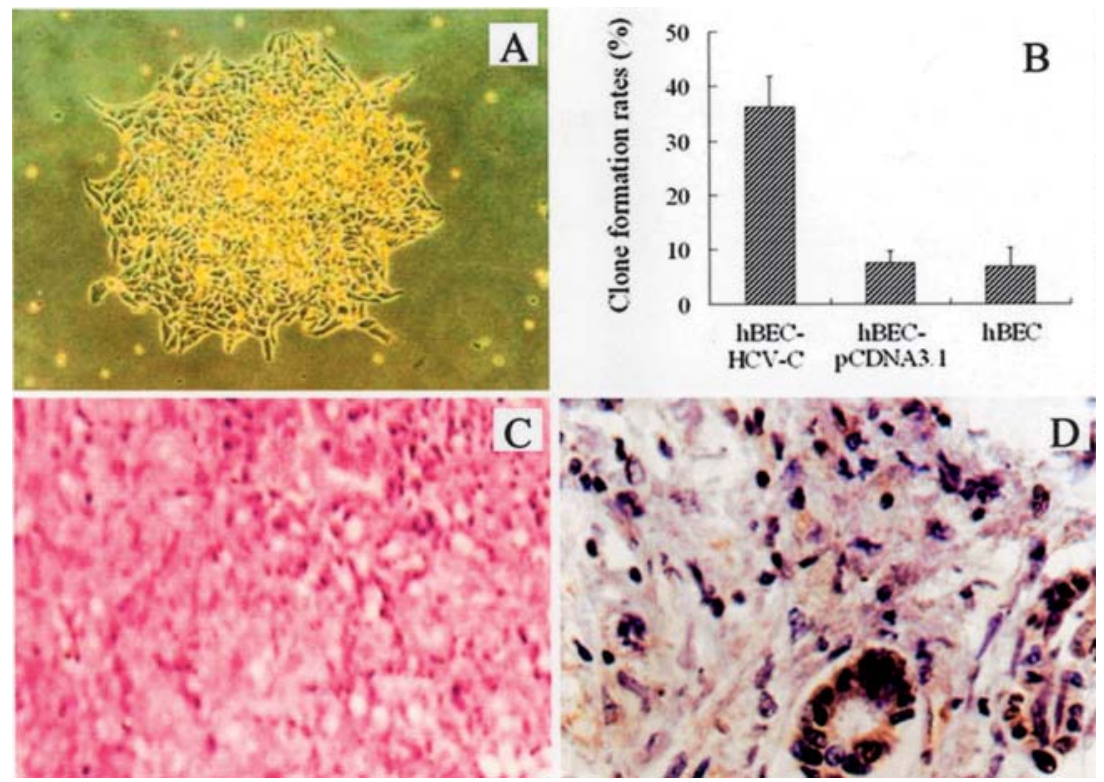

Figure 4. The clone formation and tumorigenesis of pHCV-C-transfected hBEC (hBEC-HCV-C). (A), One of hBEC-HCV-C cell clones formed in soft gel. (B), The rates of clone formation of hBEC-HCV-C cells, hBEC-pcDNA 3.1 cells and mock-transfected hBEC. (C), H\&E staining showing tumor tissues with the same pathological changes as cholangiocellular carcinoma (x200). (D), Immunohistochemical staining of tumor tissues formed by hBEC-HCV-C cells. Brown staining indicates the HCV-C positive signal, hematoxylin was used for counterstaining (x400).

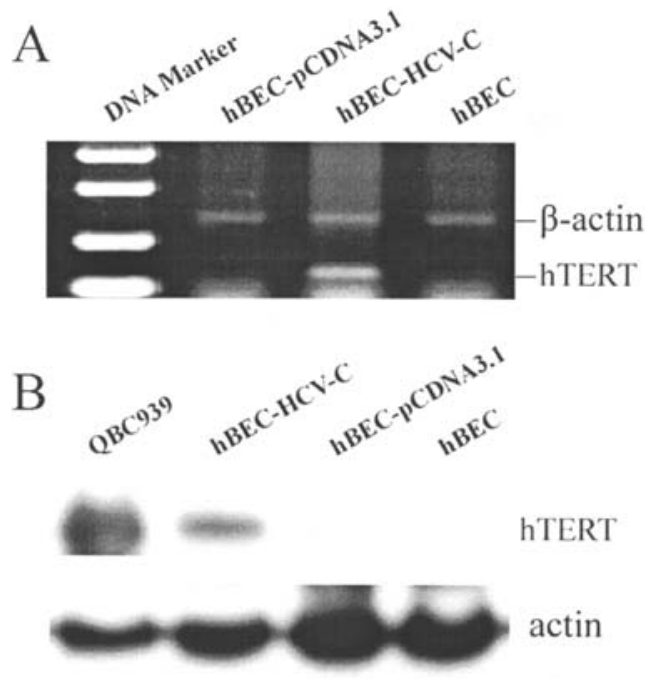

Figure 5. hTERT expression in pHCV-C transfected hBEC. (A), RT-PCR analysis of the expression of hTERT. Total cellular RNAs of hBEC-HCV-C, hBEC-pcDNA3.1 and hBEC were isolated, hTERT mRNAs were detected by RT-PCR amplification, with $B$-actin used as the internal control (hTERT, $152 \mathrm{bp}$; B-actin, $417 \mathrm{bp}$ ). (B), Western blot analysis of the expression of hTERT in hBEC-HCV-C, hBEC-pcDNA3.1 and hBEC. QBC939 used as positive control.

Immunohistochemical staining showed the expression of HCV-C in cellular plasma (Fig. 4D).

$H C V$-C up-regulated telomerase activity by up-regulating the expression of hTERT in hBEC-HCV-C cells. To investigate the mechanism of malignant transformation of $\mathrm{hBE}$ cells induced by $\mathrm{HCV}-\mathrm{C}$, we further studied the telomerase activity in hBEC-HCV-C cells. A positive telomerase activity in hBECHCV-C cells, as well as QBC939 cells, was detected, but not in hBEC-pcDNA 3.1 or mock-transfected hBE cells. The OD values of hBEC-HCV-C cells and QBC939 cells were $0.4025 \pm 0.0872$ and $0.5314 \pm 0.0967$, respectively, significantly higher than those of hBEC-pcDNA 3.1 cells $(0.0681 \pm 0.0531)$ and mock-transfected hBE cells $(0.07300 \pm 0.0621)(\mathrm{p}<0.05)$. The result suggested that HCV-C could up-regulate the telomerase activity in hBEC-HCV-C cells.

In further research, hTERT mRNA and protein were detected in hBEC-HCV-C cells by RT-PCR and Western blot analysis. However, there were almost no hTERT mRNA and protein found in hBEC-pcDNA 3.1 cells or mock-transfected hBEC (Fig. 5). These results hinted that HCV-C was able to elevate the expression of hTERT, which in turn promotes the activity of telomerase and results in cell immortalization.

\section{Discussion}

Hepatisis C virus (HCV) has been the focus of numerous previous studies in carcinogenesis. Increasing evidence suggests that HCV antigens affect multiple cellular processes and play an important role in cholangiocarcinogenesis (10-12). However, it is difficult to evaluate the functional effects of $\mathrm{HCV}$ core antigen when it is expressed together with other viral proteins.

In this research, human normal biliary tract endothelial cells were transfected with recombinant plasmid encoding $\mathrm{HCV}$ core antigen, $\mathrm{pHCV}-\mathrm{C}$, and a cell model expressing only $\mathrm{HCV}-\mathrm{C}$ core antigen (HCV-C) was established. Furthermore, the effects of HCV-C on the biological properties of the human biliary tract endothelial cells were studied. We observed morphological changes of the cells expressing HCV-C after transfection. The biological properties of the cells exhibited a dramatic alteration compared to control cells: a) majority of the cells exhibited a cholangiocarcinoma-like phenotype, consistent with the results from other studies on primary human hepatocytes $(13,14)$; b) The growth rate and colony-forming 
ability in soft agarose gels of the cells markedly increased. The ability of HCV-C to alter the proliferation properties of hBE cells was similar to the results from other studies, which examined the effects of HCV-C on the growth of the cells from other sources, such as primary human hepatocytes, mouse fibroblast cells and primary rat embryo fibroblasts (15). Collectively, it was speculated that the cells expressing HCV$\mathrm{C}$ had a tendency of malignancy conversion.

The biological properties of the cells expressing HCV-C were further investigated in nude mice by subcutaneous inoculation of the cells transfected with plasmid pHCV-C. Results showed that 7/7 nude mice, inoculated with the HCVC-positive cells, generated tumors 18 days later. Histological phenotypes of the tumors were consistent with those of cholangiocarcinoma. Our findings provide further evidence to pathomorphology that $\mathrm{HCV}-\mathrm{C}$-expression leads to the malignant conversion of the human normal biliary tract endothelium-derived cell lines (hBEC).

Immortalization of human cells requires the reactivation of the telomere-lengthing enzyme, telomerase (16-18), as the maintenance of telomeres is required for cells to escape from replicative senescence and proliferate indefinitely (19-21). It is possible that the cells will be irretrievably committed to growth once the telomerase is activated (22). In our research, hBE cells transfected with HCV core gene exhibited distinct telomerase activity as observed with positive control HeLa cells and cholangiocarcinoma QBC939 cells. However, telomerase activity was not detected in the pcDNA3.1 transfected hBE cells. These results suggested that telomerase was reactivated in $\mathrm{pHCV}-\mathrm{C}$ transfected human $\mathrm{hBE}$ cells and resulted in immortalization.

Human telomerase is composed of human telomerase RNA, telomerase-associated protein 1 , and human telomerase reverse transcriptase (hTERT) $(23,24)$. hTERT is believed to be a major determinant of telomerase activity. In our study, both hTERT protein and mRNA expression was up-regulated in hBE cells transfected with pHCV-C, but not in pCDNA3.1 or mock-transfected hBE cells, as also observed in various cancer cells (25-27).

In conclusion, $\mathrm{HCV}-\mathrm{C}$ was able to induce the malignant transformation of human biliary epithelial cells, and the activation of telomerase may be involved in this process. However, cholangiocarcinogenesis, as the genesis of other malignant tumors, is a multi-step process. The activation of telomerase may be only one of the mechanisms in which $\mathrm{HCV}$ core protein induces malignant transformation of human biliary epithelial cells. Further investigation on the other molecular mechanisms resulting in malignant conversion of the cells may help to understand the pathogenesis of cholangio-carcinoma. In addition, target inhibition of core protein function may offer new therapeutic ways for cholangiocarcinoma.

\section{Acknowledgements}

This study was supported by grants from Hi-tech Research and Development Program of P.R. China (No. 2002AA214061), Research and Development Grant of Guangdong Provine (No. 2003A031700), and Natural Science Foundation of Guangdong Province (No. 5300675).

\section{References}

1. Fukutomi T, Zhou Y, Kawai S, Eguchi H, Wands JR and Li J: Hepatitis $\mathrm{C}$ virus core protein stimulates hepatocyte growth: correlation with up-regulation of wnt-1 expression. Hepatology 41: 1096-1105, 2005.

2. Moriya K, Fujie H, Shintani Y, Yotsuyanagi H, Tsutsumi T, Ishibashi K, Matsuura Y, Kimura S, Miyamura T and Koike K: The core protein of hepatitis $C$ virus induce hepatocellular carcinoma in transgenic mice. Nature Med 4: 1065-1067, 1998.

3. Chen RF, Li ZH, Zou SQ and Chen JS: Effect of hepatitis C virus core protein on modulation of cellular proliferation and apoptosis in hilar cholangiocarcinoma. Hepatobiliary Pancreac Dis Int 4: 71-74, 2005.

4. Steffen M, Zuehlke I and Scherdin U: Motility factors identified in supernatants of human cholangiocarcinoma cell lines. Int J Oncol 18: 1107-1112, 2001.

5. Wu GS, Zou SQ, Liu ZR and Wang DY: Bile from a patient with anomalous pancreaticobiliary ductal union promotes the proliferation of human cholangiocarcinoma cells via COX-2 pathway. World J Gastro 9: 1094-1097, 2003.

6. Yamagiwa Y, Meng F and Patel T: Interleukin-6 decreases senescence and increases telomerase activity in malignant human cholangiocytes. Life Sci 78: 2494-2502, 2006.

7. Cruickshank SM, Southgate J, Selby PJ and Trejdosiewicz LK: Expression and cytokine regulation of immune recognition elements by normal human biliary epithelial and established liver cell lines in vitro. J Hepatol 29: 550-558, 1998.

8. Tang QB, Sun HW and Zou SQ: The study of methylation inhibitor 5-aza-2-deoxycytidine inhibitory effect on bile duct cancer cell line in vivo and in vitro. Hepatobiliary Pancreac Dis Int 3: 151-154, 2004.

9. Hiramatsu N, Dash S and Gerber MA: HCV cDNA transfection to HepG2 cells. J Viral Hepat 4: 61-67, 1997.

10. Yamamoto S, Kubo S, Hai S, Uenishi T, Yamamoto T, Shuto T, Takemura S, Tanaka H, Yamazaki O, Hirohashi K and Tanaka T: Hepatitis $C$ virus infection as a likely etiology of intrahepatic cholangiocarcinoma. Cancer Sci 95: 592-595, 2004.

11. Polizos A, Kelekis N, Sinani C, Patsiaoura K, Papadamou G and Dalekos GN: Advanced intrahepatic cholangiocarcinoma in hepatitis $C$ virus-related decompensated cirrhosis: case report and review of the literature. Eur J Gastroenterol Hepatol 15: 331-334, 2003.

12. Nagano K, Fukuda Y, Nakano I, Katano Y, Toyoda H, Ebata M, Morita K, Yokozaki S, Takeuchi M, Hayashi K, Takeda S, Nonami T and Hayakawa T: A case of the development of two hepatocellular carcinomas and a cholangiocarcinoma with cirrhosis after elimination of serum hepatitis C virus RNA with interferon therapy. Hepatogastroenterology 47: 1436-1438, 2000.

13. Ray RB, Meyer K and Ray R: Hepatitis $C$ virus core protein promoters immortalization of primary hepatocytes. Virology 271: 197-204, 2000.

14. Tsuchihara K, Hijikata M, Fukuda K, Kuroki T, Yamamoto N and Shimotohno K: Hepatitis $\mathrm{C}$ virus core protein regulates cell growth and signal transduction pathway transmitting growth stimuli. Virology 258: 100-107, 1999.

15. Ray RB, Lagging LM, Meyer K and Ray R: Hepatitis C virus core protein cooperates with ras and transforms primary rat embryo fibroblasts to tumorigenic phenotype. J Virol 70: 4438-4443, 1996.

16. Hastie ND, Dempster M, Dunlop MG, Thompson AM, Green DK and Allshire RC: Telomere reduction in human colorectal carcinoma and with ageing. Nature 346: 866-888, 1990.

17. Lundblad V: Telomeres: taking the measure. Nature 423: 926-927, 2003.

18. Kumazaki T, Hiyama K, Takahashi T, Omatsu H, Tanimoto K, Noguchi T, Hiyama E, Mitsui Y and Nishiyama M: Differential gene expressions during immortalization of normal human fibroblasts and endothelial cells transfected with human telomerase reverse transcriptase gene. Int J Oncol 24: 1435$1442,2004$.

19. Satyanarayana A, Manns MP and Rudolph KL: Telomeres and telomerase: a dual role in hepatocarcinogenesis. Hepatology 40: 276-283, 2004.

20. Rha SY, Jeung HC, Yang WI, Kim JJ, Oh TJ, An SW and Chung HC: Alteration of hTERT full-length variant expression level showed different gene expression profiles and genomic copy number changes in breast cancer. Oncol Rep 15: 749-755, 2006. 
21. Ozaki S, Harada K, Sanzen T, Watanabe K, Tsui W and Nakanuma Y: In situ nucleic acid detection of human telomerase in intrahepatic cholangiocarcinoma and its preneoplastic lesion. Hepatology 30: 914-919, 1999.

22. Iki K, Tsujiuchi T, Majima T, Sakitani H, Tsutsumi M, Takahama M, Yoshimoto M, Nakae D, Tsunoda T and Konishi Y: Increased telomerase activity in intrahepatic cholangiocellular carcinomas induced by $\mathrm{N}$-nitrosobis(2-oxopropyl)amine in hamsters. Cancer Lett 131: 185-190, 1998.

23. Ito Y, Yoshida H, Tomoda C, Uruno T, Takamura Y, Miya A, Kobayashi K, Matsuzuka F, Kuma K and Miyauchi A: Telomerase activity in thyroid neoplasms evaluated by the expression of human telomerase reverse transcriptase (hTERT). Anticancer Res 25: 509-514, 2005.

24. Boldrini L, Faviana P, Gisfredi S, Donati V, Zucconi Y, Ursino S, Simi P, Baldinotti F, Berti P, Galleri D, Materazzi G, Basolo F, Miccoli P, Pingitore R and Fontanini G: Regulation of telomerase and its hTERT messenger in colorectal cancer. Oncol Rep 11: 395-400, 2004.
25. Dalerba P, Guiducci C, Poliani PL, Cifola I, Parenza M, Frattini M, Gallino G, Carnevali I, Giulio I, Andreola S, Lombardo C, Rivoltini L, Schweighoffer T, Belli F, Colombo MP, Parmiani G and Castelli C: Reconstitution of human telomerase reverse transcriptase expression rescues colorectal carcinoma cells from in vitro senescence: evidence against immortality as a constitutive trait of tumor cells. Cancer Res 65: 2321-2329, 2005.

26. Kim HR, Christensen R, Park NH, Sapp P, Kang MK and Park NH: Elevated expression of hTERT is associated with dysplastic cell transformation during human oral carcinogenesis in situ. Clin Cancer Res 7: 3079-3086, 2001.

27. Shibuya K, Fujisawa T, Hoshino H, Baba M, Saitoh Y, Iizasa T, Sekine Y, Suzuki M, Hiroshima K and Ohwada H: Increased telomerase activity and elevated hTERT mRNA expression during multistage carcinogenesis of squamous cell carcinoma of the lung. Cancer 92: 849-855, 2001. 